\title{
Face Recognition using Active Appearance and Type-2 Fuzzy Classifier
}

\section{Brammya G}

Department of Applied Electronics, Arunachala college of engineering Nagercoil, Tamil Nadu, India

brammyagece@gmail.com
Suki Antely A

Department of Control and Instrumentation Engineering, St.Xavier's Catholic College of Engineering

Kanyakumari, Tamil Nadu, India

sukiantly@gmail.com

\begin{abstract}
Face recognition in unrestrained surroundings has turn out to be more and more prevalent in numerous applications, namely, intelligent visual surveillance, immigration automated clearance system and identity verification systems. The conventional pipeline of a contemporary face recognition system usually consists of face alignment, face detection, feature classification, and representation. In this paper, the input images for face recognition are subjected to feature extraction using Active Appearance Model (AAM). In addition, Type 2- Fuzzy classifier is adopted for classifying the images. Moreover, the proposed scheme is compared with Neural Network, k-NN (Nearest Neighbor) and Type 1-Fuzzy classifiers and the results are obtained.
\end{abstract}

Keywords: Face recognition, Active Appearance Model, Type 2- Fuzzy classifier, Neural Network.

\section{Introduction}

Face recognition has an extensive assortment of real-world applications, and has been broadly analyzed from mid-1990s [1] [2]. Together with the development of machine learning and computer vision methods, the robustness of face recognition schemes has been considerably boosted [3]. In the meantime, additional demanding databases are constructed with the intention to authenticate the presentation of face recognition systems in practical circumstances. Occlusion remains as one of the major challenging problems, which generally subsists in realistic state of affairs [4]. The most important complicatedness of the occlusion dilemma [5] is that occlusions can take place in a variety of changeable facial areas and shapes [6, 7, 8, 9]. When wide-ranging studies have been performed to deal with this dilemma, insufficient attempts have been remunerated to deal with the problem when occlusions come about in the training data. The major widespread approach which manages with the occlusion trouble is to partition a face image into non-overlapping patches that are subsequently programmed by robust local descriptors. The improvement of such an approach is that the entire of the local features are equally autonomous so that the occluded component will not have an effect on erstwhile areas. Numerous conventional robust face illustrations are dependent on such an approach. The accomplishment of these techniques lies on a huge amount of training illustrations so as to offer copious varieties of local patterns. On the other hand, gathering a huge quantity of clean images for training is not achievable in numerous practical scenarios, that is also recognized as the data deficiency crisis. Traditional approaches deal with the crisis by means of kernel prototype similarities [10]; hybrid classifiers [11], sparse coding with manifold learning [38], etc. [19] mostly focuses the unhinged trouble among the counts of negative and positive samples.

For the group of hand-crafted local features, characteristic illustrations are Gabor [8], LBP [13], and SIFT [12]. Such local features have attained much excellent presentation for face recognition, texture classification, and object recognition. Particularly, an uncomplicated but very dominant local descriptor, known as Local Binary Pattern (LBP) was also introduced which is one of the most excellent texture descriptors and has been extensively adopted in a variety of applications, like face recognition and textures classification [13].

Traditional local face depiction techniques [14, 15], contribute to an imperfection that the extracted features could not conserve their spatial order when distinguishing to the holistic techniques $[16,17,18]$. 
A hybrid scheme is implemented to group local features into a holistic demonstration depending on their spatial locations [19]. Nevertheless, such a framework is vulnerable to occlusions as unpredictable local features are exploited in the concluding representation.

The paper contributes type 2 -fuzzy classifier for face recognition systems. Also, AAM scheme is adopted for extracting the features of the image. Moreover, the implemented model is distinguished with NN, k-NN and Type 1-fuzzy classifiers for validating the computation of the proposed method. The paper is organized as follows. Section II analyses the related works and reviews done under this topic. Section III describes the face recognition architecture and section IV demonstrates the results and discussions. Finally, section V concludes the paper.

\section{Literature Review}

\subsection{Related works}

In 2017, Yang et al. [1] has introduced a scheme to deal with the double-occlusion crisis with a restricted quantity of training data by means of a unified structure known as subclass pooling. A face image was partitioned into ordered subclasses based on their spatial locations. Moreover, a fuzzy max-pooling scheme was suggested to restrain unreliable local characteristics from occluded areas. The concluding average-pooling can improve the robustness by routinely weighting on all the subclasses. The suggested scheme was computed on two face recognition benchmarks. Investigational outcomes propose that this technique leads to a extraordinary margin of computational gain over the benchmark systems.

In 2017, Yan et al. [2] has established a novel technique for pose-invariant 3D face recognition to deal with noteworthy pose distinctions. For pose registration and estimation, a coarse-to-fine approach was implemented to identify landmarks underneath large yaw variations. Candidate landmarks were identified at the coarse search step, by means of HK curvature investigation and subdivided based on a facial geometrical structure-dependent classification approach. Candidate landmarks were detected and labelled by distinguishing with a Facial Landmark design at the fine search step. Experimentations executed on the UND/FRGC v2.0 and Bosphorus databases demonstrate that this technique has high robustness and precision to pose variations.

In 2017, Jie et al. [3] has presented a robust local descriptor for identifying the face recognitions. It includes two mechanisms, one dependent on a local binary pattern (LBP) and another one dependent onthe shearlet-decomposition. Shearlets could entirely scrutinize the singular formations of piecewise smooth images that was constructive as irregular structures and singularities hold valuable information in an original image. In addition, LBP was effectual for illustrating the edges obtained by shearlets while when the images enclose greater level of noise. Investigational outcomes by means of the Face Recognition Grand Challenge dataset demonstrate that the suggested local descriptor considerably outperforms numerous extensively adopted features.

In 2017, Jiang et al. [4] has implemented a scheme which efficiently extend the training dataset that improves the pose variance, forces of misalignment, partial occlusions and illumination variations, in addition to the overfitting throughout training. The presentation of every data augmentation technique was scrutinized on the Multi-PIE database. In addition, assessments of these techniques were carried out on YTF, IJB-A and LFW databases. Investigational results illustrate that the suggested technique can significantly develop the face recognition presentation.

In 2017, Feilong et al. [5] has established a robust face recognition scheme by using a projection matrix and a class-specific dictionary learning. Initially, the training data were disintegrated into sparse error matrix, non-class-specific dictionary, and class-specific dictionary. Subsequently, for facilitating and testing the corrupted test data, they were projected onto their equivalent original subspace, and a projection matrix among the original class-specific dictionary and the training data is learned. Wideranging researches were accomplished on publicly present data sets demonstrate that the suggested algorithm offers improved results than some traditional techniques.

\subsection{Review}

Table 1 shows the methods, features and challenges of conventional techniques based on face recognition systems. At first, Fuzzy max-pooling scheme was suggested in [1] that offers better results for data insufficiency problem and it also avoids mismatches effectively. However, SCP scheme was not generalised to other applications. Moreover, HK classification was adopted in [2] that provide increased robustness with better accuracy, but the regions of nose tip and nasion were not occluded. Similarly, Deep learning was suggested in [3] that reduces the noise power with increased precision. However, it 
was complex due to challenging process. In addition, Deep Convolution Neural Networks (DCNN) was implemented in [4] that provide increased size of the training set and it also improves the accuracy. Anyhow, there was no contemplation on illumination problems. Finally, Sparse representation-based classification (SRC) was proposed in [5], which eliminates the sparse corruptions and offers better effectiveness and robustness, however, it requires more computation time. These above mentioned challenges are considered for motivating the improvement of face recognition systems.

Table 1. Review on the traditional face recognition systems

\begin{tabular}{|c|c|c|c|}
\hline $\begin{array}{l}\text { Author } \\
\text { [citation] }\end{array}$ & $\begin{array}{l}\text { Adopted } \\
\text { methodology }\end{array}$ & Features & Challenges \\
\hline Yang et al. [1] & $\begin{array}{l}\text { Fuzzy max-pooling } \\
\text { scheme }\end{array}$ & $\begin{array}{l}\text { * Offers better results for data } \\
\text { insufficiency problem } \\
\text { * Effectively avoid mismatches }\end{array}$ & $\begin{array}{l}\text { * SCP scheme was not generalised to } \\
\text { other applications }\end{array}$ \\
\hline Yan et al. [2] & HK classification & $\begin{array}{l}\text { Increased robustness } \\
* \text { Offers better accuracy }\end{array}$ & $\begin{array}{l}\text { * Regions of nose tip and nasion were } \\
\text { not occluded }\end{array}$ \\
\hline Jie et al. [3] & Deep learning & $\begin{array}{l}* \text { Reduced noise power } \\
* \text { Increased precision }\end{array}$ & * Complex due to challenging process \\
\hline $\begin{array}{l}\text { Jiang et } a l \text {. } \\
\text { [4] }\end{array}$ & DCNN & $\begin{array}{l}* \text { Increased size of the training set } \\
* \text { Improve the accuracy }\end{array}$ & $\begin{array}{l}\text { * No contemplation on illumination } \\
\text { problems }\end{array}$ \\
\hline $\begin{array}{l}\text { Feilong et } a l \text {. } \\
\text { [5] }\end{array}$ & $\mathrm{SRC}$ & $\begin{array}{l}\text { *liminates the sparse corruptions } \\
\text { Better effectiveness and robustness }\end{array}$ & * More computation time \\
\hline
\end{tabular}

\section{Face recognition Architecture}

The overall architecture of the proposed face recognition model is demonstrated by Fig. 1. Initially, the samples of face images are given as input for extracting the features. For extracting the features, AAM technique is adopted, which could extract the features in an optimal way. Moreover, for classifying the images, type 1-classifier is exploited, which classifies the image and offers the recognized versions of face samples.

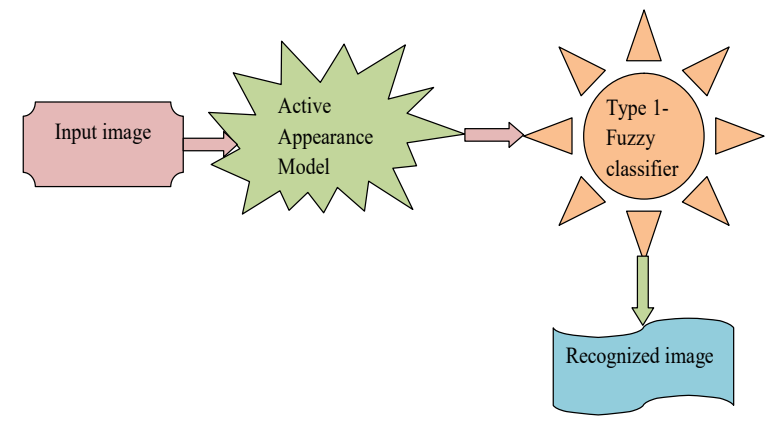

Fig. 1. Overall framework of the implemented model

\subsection{Active Appearence model}

The models are produced by merging a representation of shape dissimilarity with a representation of the appearance changes in a shape-normalised outline [22]. Moreover, a training set of labelled images are necessary, in which the landmark points are indicated on all illustration face at key positions to frame the major features. If such a set is provided, statistical representation of shape variations have to be created. The points that are labelled on a single face demonstrates the shape of that certain face. Further, the entire sets of points are aligned into a general co-ordinate frame and it indicates every by a vector $^{y}$, and subsequently, a principal component analysis (PCA) is applied to the data. Illustrations can consequently be evaluated by Eq. (1), in which ${ }^{\bar{y}}$ denotes the mean shape, $a_{s}$ refers to a group of shape parameters and $Q_{s}$ indicates a group of orthogonal modes of shape alterations.

$$
\mathrm{y}=\overline{\mathrm{y}}+\mathrm{Q}_{\mathrm{S}} \mathrm{a}_{\mathrm{S}}
$$

For constructing a numerical representation of the grey-level appearance every illustrations of the image are warped, and thus its control points equal the mean shape. In the grey level information $h_{i m}$ are 
sampled from the shape-normalised image over the area enclosed by the mean shape. For reducing the consequence of global lighting disparity, this vector is normalized obtaining $h$. On substituting PCA to this data a linear model was obtained as shown by Eq. (2), in which $\bar{h}$ indicates the mean normalised grey-level vector, $\mathrm{Q}_{\mathrm{h}}$ denotes a group of orthogonal modes of grey-level variation and $a_{h}$ refers to a group of grey-level model parameters.

$$
\mathrm{h}=\overline{\mathrm{h}}+\mathrm{Q}_{\mathrm{h}} \mathrm{a}_{\mathrm{h}}
$$

The appearance and shape of any illustration can accordingly be summarised by the vectors $a_{s}$ and $a_{h}$. As there may perhaps be connections among the shape and grey-level variations, PCA is integrated to the data. For every illustration, a concatenated vector is generated as given by Eq. (3), in which $R_{S}$ is a diagonal matrix of weights for every shape constraints, permitting for the variations in units among the grey and shape designs. Moreover, PCA is applied on these vectors, which can be indicated as given by Eq. (4), in which $c$ denotes a vector of appearance constraints and $P$ are the eigenvectors of _ $a$ controlling both the shape and grey-levels of the representation. As the grey-model and shape parameters contain zero mean, _c too has a zero mean.

$$
\begin{aligned}
& \mathrm{a}=\left(\begin{array}{l}
\mathrm{R}_{\mathrm{s}} \mathrm{a}_{\mathrm{s}} \\
\mathrm{a}_{\mathrm{h}}
\end{array}\right)=\left(\begin{array}{ll}
\mathrm{R}_{\mathrm{s}} \mathrm{Q}_{\mathrm{s}}^{\mathrm{T}}(\mathrm{y} & \overline{\mathrm{y}}) \\
\mathrm{Q}_{\mathrm{h}}^{\mathrm{T}}(\mathrm{h} & \overline{\mathrm{h}})
\end{array}\right) \\
& \mathrm{a}=\mathrm{Pc}
\end{aligned}
$$

Thus the features are extracted for the given image samples for face recognition.

\subsection{Type -2 Fuzzy Modelling Sytem (FLS)}

Type-2 FLS [23] including $n$ inputs $\mathrm{p}_{1} \in \mathrm{X}_{1} \ldots . ., \mathrm{p}_{\mathrm{n}} \in \mathrm{X}_{\mathrm{n}}$ and a single output $\mathrm{q} \in \mathrm{Y}$ Multiple Input Single Output (MISO), on assuming M rules, the $1^{\text {th }}$ rule in the type- 2 FLS can be indicated as given by Eq. (5), in which the $\mathrm{F}$ 's and $\mathrm{H}$ are suitable fuzzy sets for every rule.

$$
\mathrm{V}^{1}: \text { If } \mathrm{p}_{1} \text { is } \widetilde{\mathrm{F}}_{1}^{1} \text { and } \ldots \text { and } \widetilde{F}_{n}^{1} \text {, then } q \text { is } \widetilde{\mathrm{H}}^{1} \quad \mathrm{l}=1,2 \ldots \mathrm{M}
$$

In the type-2 FLS, the inference engine merges the rules and provides a mapping from input type- 2 FLS to output type-2 FLS. It is essential to calculate the meet intersections $\partial$ and the join unions $U$, in addition to the comprehensive sup-star compositions of type- 2 associations. If $\widetilde{\mathrm{F}}_{1}^{1} \times . . \times \widetilde{\mathrm{F}}_{\mathrm{n}}^{1}=\widetilde{\mathrm{B}}^{1}$; subsequently Eq. (5) can be modified as given by Eq. (6), where $\mathrm{V}^{1}$ is demonstrated by the membership function as shown in Eq. (7) and Eq. (8). Eq. (8) can be further modified as specified by Eq. (9)

$$
\begin{gathered}
V^{1}: \widetilde{\mathrm{F}}_{1}^{1} \times \ldots \times \widetilde{\mathrm{F}}_{\mathrm{n}}^{1} \rightarrow \widetilde{\mathrm{H}}^{1}=\widetilde{\mathrm{B}}^{1} \rightarrow \widetilde{\mathrm{H}}^{1} \quad \mathrm{l}=1,2 \ldots \mathrm{M} \\
\alpha_{\mathrm{V}^{1}}(\mathrm{p}, \mathrm{q})=\alpha_{\mathrm{V}^{1}}\left(\mathrm{p}_{1} \ldots \mathrm{p}_{\mathrm{n}}, \mathrm{q}\right) \\
\alpha_{\mathrm{V}^{1}}(\mathrm{p}, \mathrm{q})=\alpha_{\widetilde{\mathrm{B}}^{1} \rightarrow \widetilde{\mathrm{H}}^{1}}(\mathrm{p}, \mathrm{q}) \\
\alpha_{\mathrm{V}^{1}}(\mathrm{p}, \mathrm{q})=\alpha_{\widetilde{\mathrm{B}}^{1} \rightarrow \widetilde{\mathrm{H}}^{1}}(\mathrm{p}, \mathrm{q})=\alpha_{\widetilde{\mathrm{F}}_{1}^{1}}\left(\mathrm{p}_{1}\right) \sqcap \ldots \sqcap \alpha_{\widetilde{\mathrm{F}}_{\mathrm{n}}^{1}}\left(\mathrm{p}_{\mathrm{n}}\right) \sqcap \alpha_{\widetilde{\mathrm{H}}^{1}}(\mathrm{q})=\left[\prod_{\mathrm{i}=1}^{\mathrm{n}} \alpha_{\widetilde{\mathrm{F}}_{1}^{1}}\left(\mathrm{p}_{\mathrm{n}}\right)\right]=\Pi \alpha_{\widetilde{\mathrm{H}}^{1}}(\mathrm{q})
\end{gathered}
$$

Usually, the $n$-dimensional input to $V^{1}$ is specified by the type-2 FLS, $\widetilde{B}_{p}$ whose membership function is indicated by Eq. (10), in which $\tilde{X}_{i}(i=1,2 \ldots n)$ denotes the labels of the fuzzy sets defining the inputs.

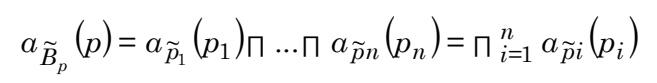

Thus the given images are classified using Type 2-fuzzy classifier.

\section{Results and Discussion}

The proposed type 1-fyzzy classifier was experimented in MATLAB and the results were obtained. The experimentation was done using benchmark database. Moreover, the suggested method was compared with conventional algorithms such as NN [21], k-NN [20], type 1-fuzzy classifiers [22] and the results were obtained.

\subsection{Performance Analysis}

The proposed type 2-fuzzy class for face recognition was compared with various conventional algorithms and the results were obtained. From Fig. 2(a), the accuracy for the proposed method for $10^{\text {th }}$ learning percentage was obtained, which is $14.7 \%$ better than $\mathrm{NN}, 9.31 \%$ better than $\mathrm{k}-\mathrm{NN}$ and $9 \%$ better than 
type 1 -fuzzy schemes. Similarly, for $20^{\text {th }}$ learning percentage, the accuracy of the suggested scheme is $12.17 \%$ superior to NN, $9.29 \%$ superior to $\mathrm{k}-\mathrm{NN}$ and $6.9 \%$ superior to type 1 -fuzzy methods. Also, for 30 th learning percentage the implemented technique is $12.34 \%$ better than NN, $10.9 \%$ better than $\mathrm{k}-\mathrm{NN}$ and $6.19 \%$ better than type 1 -fuzzy schemes. Moreover, for $40^{\text {th }}$ learning percentage, the accuracy of the presented scheme is $13.01 \%$ superior to $\mathrm{NN}, 9.45 \%$ superior to $\mathrm{k}-\mathrm{NN}$ and $6.24 \%$ superior to type 1 -fuzzy methods.

Similarly, from Fig. 2(b), the sensitivity of the proposed method was attained, where the implemented method is $15 \%$ superior to NN, $9.52 \%$ superior to k-NN and $8.35 \%$ superior to type 1 -fuzzy schemes. Similarly, for 20th learning percentage, the sensitivity of the suggested scheme is $10.07 \%$ better than NN, 8.41\% better than $\mathrm{k}-\mathrm{NN}$ and $10.76 \%$ better than type 1 -fuzzy methods. Also, for $30^{\text {th }}$ learning percentage the implemented technique is $15.53 \%$ superior to NN, 9.49\% superior to $\mathrm{k}$-NN and $10.65 \%$ superior to type 1 -fuzzy schemes. Moreover, for $40^{\text {th }}$ learning percentage, the sensitivity of the presented scheme is $14.95 \%$ better than NN, $12.58 \%$ better than $\mathrm{k}-\mathrm{NN}$ and $10.35 \%$ better than type 1 fuzzy methods. Moreover, from Fig. 2(c), the specificity for the proposed method for $10^{\text {th }}$ learning percentage was specified, which is $16.48 \%$ better than NN, $9.31 \%$ better than k-NN and $5.7 \%$ better than type 1-fuzzy schemes. Similarly, for $20^{\text {th }}$ learning percentage, the specificity of the suggested scheme is $12 \%$ superior to $\mathrm{NN}, 11.52 \%$ superior to $\mathrm{k}-\mathrm{NN}$ and $6.8 \%$ superior to type 1 -fuzzy methods. Also, for 30 th learning percentage the implemented technique is $12.30 \%$ better than NN, $8.54 \%$ better than $\mathrm{k}$-NN and $6.2 \%$ better than type 1 -fuzzy schemes. Moreover, for $40^{\text {th }}$ learning percentage, the specificity of the presented scheme is $12.82 \%$ superior to $\mathrm{NN}, 9.3 \%$ superior to $\mathrm{k}-\mathrm{NN}$ and $7 \%$ superior to type 1 -fuzzy methods. In addition, from Fig. 2(d), the precision of the proposed method was attained, where the implemented method is $12.6 \%$ superior to NN, $6.76 \%$ superior to $\mathrm{k}-\mathrm{NN}$ and $7.9 \%$ superior to type 1 -fuzzy schemes. Similarly, for $20^{\text {th }}$ learning percentage, the suggested scheme is $12.73 \%$ better than NN, $9.99 \%$ better than k-NN and 9.1\% better than type 1-fuzzy methods. Also, for 30th learning percentage the implemented technique is $10.05 \%$ superior to NN, $7.87 \%$ superior to $\mathrm{k}-\mathrm{NN}$ and $2.89 \%$ superior to type 1 fuzzy schemes. Moreover, for $40^{\text {th }}$ learning percentage, the precision of the presented scheme is $9.54 \%$ better than NN, $4.84 \%$ better than $\mathrm{k}-\mathrm{NN}$ and $1.41 \%$ better than type 1-fuzzy methods. Similarly, from Fig. 2(e), the presented method regarding FPR for $10^{\text {th }}$ learning percentage was $10 \%$ better than NN, $0.54 \%$ better than $\mathrm{k}-\mathrm{NN}$ and $1.2 \%$ better than type 1 -fuzzy schemes. Similarly, for $20^{\text {th }}$ learning percentage, the FPR of the suggested scheme is $20.14 \%$ superior to NN, 2.67\% superior to k-NN and $2.3 \%$ superior to type 1-fuzzy methods. Also, for $30^{\text {th }}$ learning percentage the implemented technique is $17.17 \%$ better than NN, 5.24\% better than k-NN and $2.9 \%$ better than type 1-fuzzy schemes. Moreover, for $40^{\text {th }}$ learning percentage, the $\mathrm{FPR}$ of the presented scheme is $20.24 \%$ superior to NN, $14.41 \%$ superior to k-NN and 3.43\% superior to type 1-fuzzy methods. Also, from Fig. 2(f), the presented technique regarding FNR is $7.96 \%$ superior to NN, $4.62 \%$ superior to $\mathrm{k}-\mathrm{NN}$ and $2.58 \%$ superior to type 1 fuzzy schemes. Similarly, for $20^{\text {th }}$ learning percentage, the FNR of the suggested scheme is $9 \%$ better than NN, 2.63\% better than k-NN and 3.68\% better than type 1-fuzzy methods. Also, for 30th learning percentage the implemented technique is $17 \%$ superior to NN, $2.7 \%$ superior to $\mathrm{k}-\mathrm{NN}$ and $0.4 \%$ superior to type 1-fuzzy schemes. Moreover, for $40^{\text {th }}$ learning percentage, the $\mathrm{FNR}$ of the presented scheme is $18.61 \%$ better than NN, $6.09 \%$ better than $\mathrm{k}-\mathrm{NN}$ and $4.74 \%$ better than type 1 -fuzzy methods. Moreover, from Fig. 2(g), the NPV of proposed method is $7.38 \%$ better than NN, $1.84 \%$ better than $\mathrm{k}-\mathrm{NN}$ and $1.1 \%$ better than type 1-fuzzy schemes. Similarly, for $20^{\text {th }}$ learning percentage, the NPV of the suggested scheme is $8.28 \%$ superior to $\mathrm{NN}, 8.34 \%$ superior to $\mathrm{k}-\mathrm{NN}$ and $6.2 \%$ superior to type 1 -fuzzy methods. Also, for $30^{\text {th }}$ learning percentage the implemented technique is $14.78 \%$ better than NN, $10.18 \%$ better than $\mathrm{k}-\mathrm{NN}$ and $9.8 \%$ better than type 1 -fuzzy schemes. Moreover, for $40^{\text {th }}$ learning percentage, the NPV of the presented scheme is $15.69 \%$ superior to NN, $9.41 \%$ superior to $\mathrm{k}-\mathrm{NN}$ and $8.15 \%$ superior to type 1 fuzzy methods. Similarly, from Fig. 2(h), the FDR of suggested method is $6.43 \%$ superior to NN, $3.15 \%$ superior to k-NN and $1.67 \%$ superior to type 1 -fuzzy schemes. Similarly, for $20^{\text {th }}$ learning percentage, the suggested scheme is $10.4 \%$ better than NN, $8.6 \%$ better than $\mathrm{k}-\mathrm{NN}$ and $5.5 \%$ better than type 1 -fuzzy methods. Also, for $30^{\text {th }}$ learning percentage the implemented technique is $16.21 \%$ superior to NN, $4.18 \%$ superior to $\mathrm{k}-\mathrm{NN}$ and $0.47 \%$ superior to type 1-fuzzy schemes. Moreover, for $40^{\text {th }}$ learning percentage, the FDR of the presented scheme is $20.89 \%$ better than NN, 7.35\% better than k-NN and $5.9 \%$ better than type 1-fuzzy methods. Moreover, from Fig. 2(i), the implemented process for F1-score is $12.25 \%$ better than NN, 9.1\% better than k-NN and 8.16\% better than type 1-fuzzy schemes. Similarly, for $20^{\text {th }}$ learning percentage, the F1-score of the suggested scheme is $13.63 \%$ superior to NN, $9.68 \%$ superior to $\mathrm{k}-\mathrm{NN}$ and $7.62 \%$ superior to type 1 -fuzzy methods. Also, for $30^{\text {th }}$ learning percentage the implemented technique is $14.21 \%$ better than $\mathrm{NN}, 9.38 \%$ better than $\mathrm{k}-\mathrm{NN}$ and $8.24 \%$ better than type 1 -fuzzy schemes. Moreover, for $40^{\text {th }}$ learning percentage, the F1-score of the presented scheme is $11.6 \%$ superior to NN, $10.35 \%$ superior to k-NN and 14.7\% superior to type 1-fuzzy methods. Finally, from Fig. $2(\mathrm{j})$, the MCC for suggested scheme is $12.63 \%$ superior to $\mathrm{NN}, 10.03 \%$ superior to $\mathrm{k}-\mathrm{NN}$ and $9.1 \%$ 
superior to type 1-fuzzy schemes. Similarly, for $20^{\text {th }}$ learning percentage, the suggested scheme is $9.1 \%$ better than NN $7.46 \%$ better than $\mathrm{k}-\mathrm{NN}$ and $12.9 \%$ better than type 1 -fuzzy methods. Also, for $30^{\text {th }}$ learning percentage the implemented technique is $6.32 \%$ superior to $\mathrm{NN}, 4.41 \%$ superior to $\mathrm{k}-\mathrm{NN}$ and $0.7 \%$ superior to type 1-fuzzy schemes. Moreover, for $40^{\text {th }}$ learning percentage, the MCC of the presented scheme is $6.6 \%$ better than NN5.81\% better than $\mathrm{k}-\mathrm{NN}$ and $1.2 \%$ better than type 1-fuzzy methods.

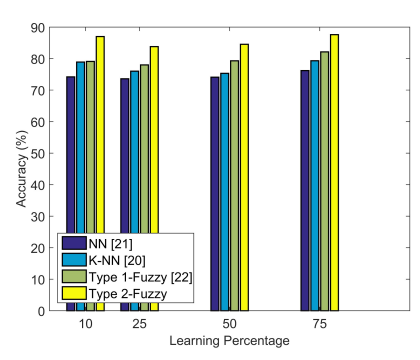

(a)

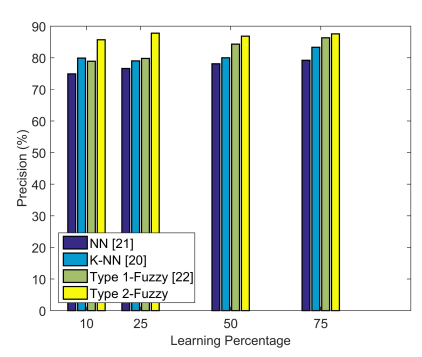

(d)

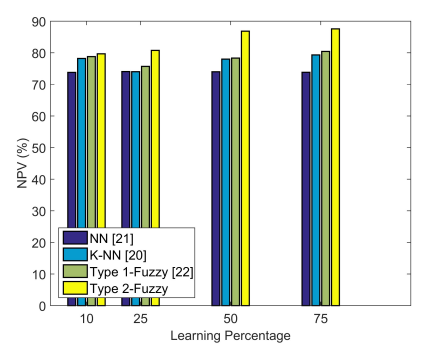

(g)

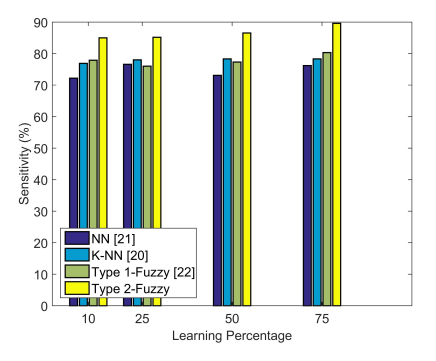

(b)

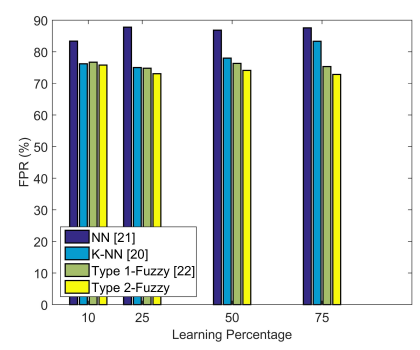

(e)

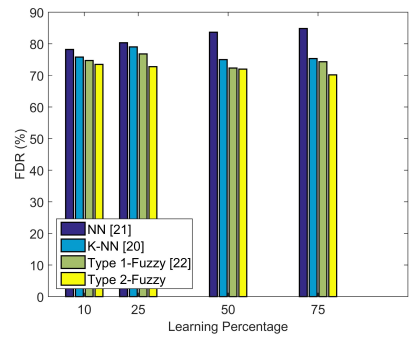

(h)

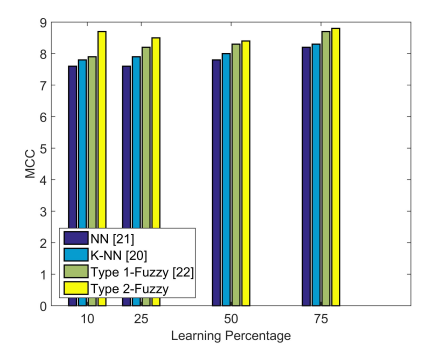

(j)

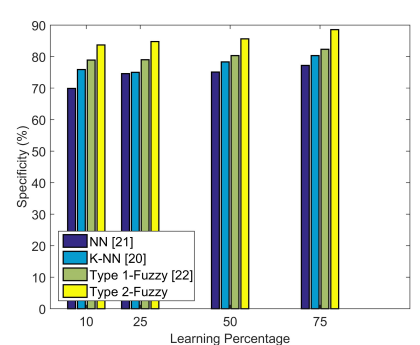

(c)

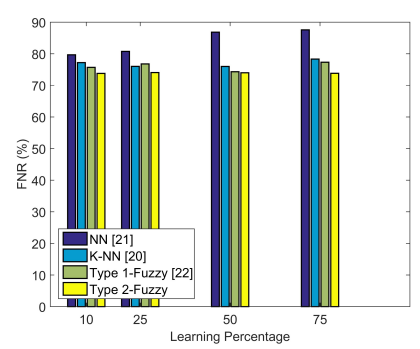

(f)

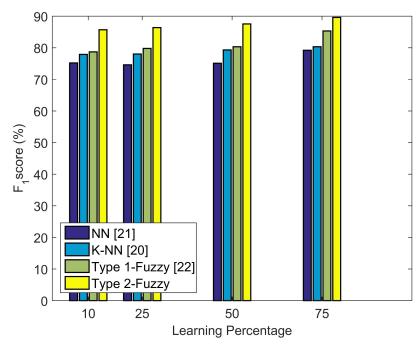

(i)

Fig. 2. Overall performance analysis for (a)Accuracy (b) Sensitivity (c) Specificity (d) Precision (e) FPR (f) FNR (g) NPV (h) FDR (i) F1-score (j)MCC

\subsection{Overall Analysis}

The implemented type 2-fuzzy class for face recognition was compared for various measures with conventional algorithms such as NN, k-NN and type 1-fuzzy classifiers. From Table II, the implemented method regarding accuracy is $14.7 \%$ better than NN, 9.31\% better than $\mathrm{k}-\mathrm{NN}$ and $9 \%$ better than type 1fuzzy schemes. Similarly, the sensitivity of the proposed scheme is $10.07 \%$ superior to NN, $8.41 \%$ superior to k-NN and $10.76 \%$ superior to type 1-fuzzy methods. Moreover, for specificity, the presented 
technique is $12.30 \%$ better than $\mathrm{NN}, 8.54 \%$ better than $\mathrm{k}-\mathrm{NN}$ and $6.2 \%$ better than type 1 -fuzzy schemes. Similarly, the suggested scheme regarding precision is $9.54 \%$ superior to NN, $4.84 \%$ superior to k-NN and $1.41 \%$ superior to type 1 -fuzzy systems. In addition, the FPR of proposed method is $20.14 \%$ better than NN, $2.67 \%$ better than $\mathrm{k}-\mathrm{NN}$ and $2.3 \%$ better than type 1-fuzzy methods. Also, the FNR of proposed scheme is $7.96 \%$ superior to $\mathrm{NN}, 4.62 \%$ superior to $\mathrm{k}-\mathrm{NN}$ and $2.58 \%$ superior to type 1 -fuzzy schemes. Moreover, the presented scheme regarding NPV is $14.78 \%$ better than NN, $10.18 \%$ better than k-NN and $9.8 \%$ better than type 1 -fuzzy schemes. In addition, the FDR of implemented method is $20.89 \%$ superior to NN, 7.35\% superior to k-NN and 5.9\% superior to type 1-fuzzy methods. Similarly, the F1-score of the proposed method is $12.25 \%$ better than NN, $9.1 \%$ better than $\mathrm{k}-\mathrm{NN}$ and $8.16 \%$ better than type 1-fuzzy schemes. Finally, the proposed scheme regarding MCC is $6.32 \%$ superior to NN, $4.41 \%$ superior to k-NN and $0.7 \%$ superior to type 1 -fuzzy techniques. Thus the overall computation of the proposed type1-fuzzy classifier was validated successfully.

Table 2. Overall Performance of the proposed design

\begin{tabular}{lrrrr}
\hline Methods & NN [21] & $k$-NN [20] & Type 1-Fuzzy & Type 2- Fuzzy \\
\hline Accuracy & 74.2 & 78.9 & 79.1 & 87 \\
Sensitivity & 76.6 & 78.01 & 76.01 & 85.18 \\
Specificity & 75.1 & 78.32 & 80.32 & 85.64 \\
Precision & 79.2 & 83.32 & 86.32 & 87.56 \\
FPR & 87.78 & 75.01 & 74.79 & 73.06 \\
FNR & 79.67 & 77.20 & 75.7 & 73.79 \\
NPV & 73.99 & 77.99 & 78.32 & 86.83 \\
FDR & 84.82 & 75.32 & 74.32 & 70.16 \\
Fiscore & 75.2 & 77.9 & 78.7 & 85.7 \\
MCC & 78.91 & 80.52 & 83.62 & 84.24 \\
\hline
\end{tabular}

\section{Conclusion}

This paper has presented type 2-fuzzy classifier for face recognition systems. Accordingly, AAM was adopted for extracting the features of the image. Moreover, the implemented model was compared with NN, k-NN and Type 1-fuzzy classifiers for validating the performance of the proposed method. From the analysis, the implemented method regarding accuracy is $14.7 \%$ better than NN, $9.31 \%$ better than $\mathrm{k}-\mathrm{NN}$ and $9 \%$ better than type 1-fuzzy schemes. Similarly, the sensitivity of the proposed scheme is $10.07 \%$ superior to $\mathrm{NN}, 8.41 \%$ superior to $\mathrm{k}-\mathrm{NN}$ and $10.76 \%$ superior to type 1-fuzzy methods. Moreover, for specificity, the presented technique is $12.30 \%$ better than $\mathrm{NN}, 8.54 \%$ better than $\mathrm{k}-\mathrm{NN}$ and $6.2 \%$ better than type 1-fuzzy schemes. Similarly, the suggested scheme regarding precision is $9.54 \%$ superior to NN, $4.84 \%$ superior to $\mathrm{k}-\mathrm{NN}$ and $1.41 \%$ superior to type 1 -fuzzy systems. In addition, the FPR of proposed method is $20.14 \%$ better than NN, $2.67 \%$ better than k-NN and $2.3 \%$ better than type 1-fuzzy methods. Thus the computational capability of the suggested type -1 fuzzy model was verified from the obtained results.

\section{Compliance with Ethical Standards}

Conflicts of interest: Authors declared that they have no conflict of interest.

Human participants: The conducted research follows the ethical standards and the authors ensured that they have not conducted any studies with human participants or animals.

\section{References}

[1] Yang Long, Fan Zhu, Ling Shao, Junwei Han, "Face recognition with a small occluded training set using spatial and statistical pooling”, Information Sciences, 31 October 2017.

[2] Yan Liang, Yun Zhang, Xian-Xian Zeng, "Pose-invariant 3D face recognition using half face", Signal Processing: Image Communication, vol. 57, pp. 84-90, September 2017.

[3] Jie Chen, Vishal M. Patel, Li Liu, Vili Kellokumpu, Rama Chellappa, "Robust local features for remote face recognition", Image and Vision Computing, vol. 64, pp. 34-46, August 2017.

[4] Jiang-Jing Lv, Xiao-Hu Shao, Jia-Shui Huang, Xiang-Dong Zhou, Xi Zhou, "Data augmentation for face recognition", Neurocomputing, vol. 230, pp. 184-196, 22 March 2017.

[5] Feilong Cao, Xinshan Feng, Jianwei Zhao, "Sparse representation for robust face recognition by dictionary decomposition", Journal of Visual Communication and Image Representation, vol. 46, pp. 260-268, July 2017.

[6] Martinez, A. and Benavente, R, "The ar face database", In CVC Technical Report, pp. 24, 1998. 
[7] 7. Phillips, P. J., Moon, H., Rizvi, S. A., and Rauss, P. J, "The feret evaluation methodology for face-recognition algorithms", IEEE Transactions on Pattern Analysis and Machine Intelligence, vol. 22, no.10, pp.1090-1104, 2000.

[8] Lee, K.-C., Ho, J., and Kriegman, D. J. "Acquiring linear subspaces for face recognition under variable lighting”, IEEE Transactions on Pattern Analysis and Machine Intelligence, vol. 27, no.5, pp.684-698, 2005.

[9] Phillips, P. J., Moon, H., Rizvi, S. A., and Rauss, P. J, "The feret evaluation methodology for face-recognition algorithms", IEEE Transactions on Pattern Analysis and Machine Intelligence, vol.22, no.10, pp.1090-1104, 2000.

[10] Klare, B. F. and Jain, A. K," Heterogeneous face recognition using kernel prototype similarities”, IEEE Transactions on Pattern Analysis and Machine Intelligence, vol. 35, no.6, pp.1410-1422, 2013.

[11] Osadchy, M., Keren, D., and Raviv, D, "Recognition using hybrid classifiers", IEEE Transactions on Pattern Analysis and Machine Intelligence, vol. 38, no.4, pp.759-771, 2016.

[12] Zhang, P., You, X., Ou, W., Chen, C. P., and Cheung, Y.-m, "Sparse discriminative multi-manifold embedding for one-sample face identification", Pattern Recognition, vol. 52, pp. 249-259, 2016.

[13] Zhu, M. and Martinez, A. M," Subclass discriminant analysis “, IEEE Transactions on Pattern Analysis and Machine Intelligence", vol. 28, no.8, pp.1274-1286, 2006.

[14] Martinez, A. M, "Recognizing imprecisely localized, partially occluded, and expression variant faces from a single sample per class", IEEE Transactions on Pattern analysis and machine intelligence, vol. 24, no.6, pp.748$763,2002 \mathrm{a}$.

[15] Tan, X., Chen, S., Zhou, Z.-H., and Zhang, F, "Recognizing partially occluded, expression variant faces from single training image per person with som and soft k-nn ensemble",IEEE Transactions on Neural Networks, vol.16, no. 4, pp.875-886, 2005.

[16] Martinez, A. M, "Recognizing imprecisely localized, partially occluded, and expression variant faces from a single sample per class", IEEE Transactions on Pattern analysis and machine intelligence, vol. 24, no.6, pp.748$763,2002 \mathrm{~b}$.

[17] Belhumeur, P. N., Hespanha, J. P., and Kriegman, D. J, "Eigenfaces vs. fisherfaces: Recognition using class specific linear projection”, IEEE Transactions on Pattern Analysis and Machine Intelligence, vol. 19, no.7, pp.711-720, 1997.

[18] Wright, J., Yang, A. Y., Ganesh, A., Sastry, S. S., and Ma, Y, "Robust face recognition via sparse representation", IEEE Transactions on Pattern Analysis and Machine Intelligence, vol. 31, no. 2, pp. 210-227, 2009.

[19] Sakoe, H. and Chiba, S, "Dynamic programming algorithm optimization for spoken word recognition. IEEE Transactions on Acoustics", Speech, and Signal Processing, vol. 26, no. 1, pp. 43-49, 1978.

[20] Zhenyun Deng, Xiaoshu Zhu, Debo Cheng, Ming Zong, Shichao Zhang, "Efficient kNN classification algorithm for big data", Neurocomputing, vol. 195, pp. 143-148, 26 June 2016.

[21] Bikesh Kumar Singh, Kesari Verma b, A.S. Thoke, "Fuzzy cluster based neural network classifier for classifying breast tumors in ultrasound images",Expert Systems With Applications, vol. 66, pp.114-123, 2016.

[22] G.J. Edwards, T.F. Cootes, and C.J. Taylor, "Face Recognition Using Active Appearance Models", Wolfson Image Analysis Unit, 1998.

[23] O. Castillo and P. Melin, Type-2 Fuzzy Logic Systems,SpringerBriefs in Computational Intelligence, 2012 